\title{
Non-linear response of coupled soil-pile-structure system under sinusoidal excitations with various frequencies
}

\author{
Mahmoud N. Hussien*, Tetsuo Tobita**, and Susumu Iai*** \\ * PhD student Dept. of Civil and Earth Resources Eng. Kyoto University (Katsura Campus, Nishikyo-ku, Kyoto, 615-8540) \\ ** Member PhD Assistant Prof. Disaster Prevention Research Institute, Kyoto University (Gokasho, Uji, Kyoto, 611-0011) \\ *** Member Dr. of Eng. Prof. Disaster Prevention Research Institute, Kyoto University (Gokasho, Uji, Kyoto, 611-0011)
}

\begin{abstract}
The non-linear response of coupled soil-pile-structure systems to seismic loading is parametrically studied in the frequency domain using two-dimensional (2D) finite elements (FE). The soil-pile interaction in three dimensions (3D) is idealized in the 2D type using soil-pile interaction springs with non-linear hysteretic load displacement relationships. The system under investigation comprises of a single degree of freedom structure supported by an end-bearing single pile founded in a homogenous sand layer over rigid rock. Comparisons with established results from the literature suggest that the adopted FE model reasonably captures the essential features of the seismic response of the coupled soil-pile-structure system. Numerical results demonstrate the strong influence on the effective natural period of the foundation properties. The effect of non-linear soil behavior and soil profile as well as the frequency content of excitation on both kinematic and inertial interactions is illustrated. The relative contributions of kinematic and inertial interaction to the development of dynamic pile bending are clarified.
\end{abstract}

Key Words: Soil-pile-interaction, finite elements, coupled system, dynamic bending moment, seismic response

\section{Introduction}

The available procedures of seismic soil-pile-structure interaction (SSPSI) estimation include those based on simplified interactions models such as the beam on dynamic Winkler Foundation approach ${ }^{1), 2)}$, as well as those based on more rigorous $\mathrm{FEM}^{3), 4)}$ or $\mathrm{BEM}^{5),}$ 6) formulations. These methods utilize either simplified two-step methods that uncouple the structure and foundation portions ${ }^{7), 8)}$ or a fully coupled SSPSI system in a single step ${ }^{4), 9}$. The coupled 3D FE approach is most representative of the SSPSI system especially when the material nonlinearity caused by soil plasticity is included in the analysis. However, such this method, even if available, has well-known limitations when used in seismic design. This particularly true if the seismic analysis using actual or simulated ground motions is to be performed in the frequency domain. Under these conditions, SSPSI must be computed for a large number of frequencies covering the frequency content of the seismic signal.

Several investigators attempted to propose new procedures to simplify the rigorous 3D FE models. For example, Ozutsumi et al. ${ }^{10)}$ proposed a method to idealize the soil-pile interaction in $3 \mathrm{D}$ into the 2D type using soil-pile interaction springs. While the conventional spring elements used in the analysis of soil-pile interactions are embedded in the same plane of the $2 \mathrm{D} \mathrm{FE}$ analysis domain, the soil-pile interaction spring proposed by Ozutsumi et al. ${ }^{10)}$ is a spring that connect free pile elements to a 2D mesh of soil profile with non-linear hysteretic load displacement relationships.

In this paper, the non-linear response of coupled SSPSI systems under seismic loading is parametrically studied in the frequency domain using 2D FE adopting the simplified model proposed by Ozutsumi et al. ${ }^{10)}$. A 2D effective stress FE analysis, FLIP $^{11)}$, is employed to this end. The present paper focuses on identification of the fundamental frequencies that dominate the response of SSPSI system not only with linear soil properties but also with non-linear properties of soil. The effects of soil profile and non-linear soil properties on the free-field response, kinematic soil-pile interaction as well as the effective natural frequency of the coupled system are elucidated. The relative contributions of kinematic and inertial interaction to the 
development of dynamic pile bending are also clarified. Particular concern is placed on the validation of the FE model by comparing the numerical results with those found using other established approaches. The verification of the adopted model is important for practical purposes. Although validation of the current FE model is confirmed in the time domain through a comparison of the FE results with recorded centrifuge data ${ }^{12}$, verification of the model in the frequency domain is necessary.

\section{Problem Definition}

The response of a fixed base single degree of freedom (SDOF) structure to seismic loading depends basically on the structural characteristics, namely its mass $\mathrm{m}_{\mathrm{st}}$ and stiffness $\mathrm{k}_{\mathrm{st}}$ and the fixed base fundamental frequency of the structure can be given as:

$$
f_{\text {st.fixed }}=\frac{1}{2 \pi} \sqrt{\frac{k_{s t}}{m_{s t}}}
$$

If the predominant frequency of the input motion is close to the fixed base frequency, the structure displacement would be maximized relative to the displacement of the input motion.

When the structure is founded on a compliant soil, not only the structural characteristics but also soil properties will control the response of the soil-structure system leading to the definition of the effective natural frequency $\left(f_{\mathrm{SSI}}\right)$. When the predominant frequency of input motion is close to this frequency, the structure displacement $\left(\mathrm{U}_{\mathrm{s}}\right)$ will be maximized relative to the free field soil displacement $\left(\mathrm{U}_{\mathrm{ff}}\right)$.

If the structure is supported by piles, both structural characteristics and foundation (soil + pile) properties will control SSPSI response. Not only the effective natural frequency $\left(f_{\text {SSI }}\right)$ will control the response in this case but also a pseudo-natural frequency $\left(f_{\mathrm{pSSI}}\right)$ does. $f_{\mathrm{pSSI}}$ is first introduced by Rovithis et al. ${ }^{4}$ as the frequency at which the structure displacement is maximized relative to the pile-head displacement. In this article, the effects of soil profile as well as non-linear soil behavior on the aforementioned frequencies will be illustrated.

The SSPSI examined in this study comprises of an end-bearing single pile supporting a single degree of freedom (SDOF) structure founded on a homogeneous dry sand layer of thickness $(\mathrm{L}=30 \mathrm{~m})$ over rigid rock as shown in Fig. 1. The input motion is specified at bedrock level in the form of harmonic horizontal excitations. These excitations consist of sinusoidal waves of constant amplitude and varying frequency. In the analysis, the non-linear behavior of soil has been discussed by changing the amplitude of input motions.

\section{Numerical Modeling and Material Properties}

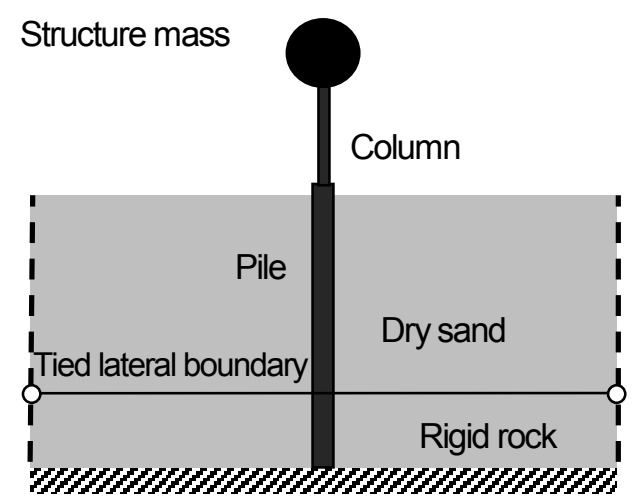

Fig. 1 A schematic view of the system under investigation

The 2D effective stress FE analysis, FLIP ${ }^{11)}$, has been used to analyze the SSPSI problem. A 30 (m) thick soil stratum was meshed with quad plane elements. The dimension of soil elements limits the value of the highest frequency which is transferable. For that, the vertical dimension of a soil element follows ${ }^{13)}$ :

$$
h \leq \frac{1}{5} \mathrm{~V}_{s}^{\prime} / f_{\max }
$$

where $f_{\max }$ is the highest frequency needed in the analysis, $f_{\max }=$ $10 \mathrm{~Hz}$ and $\mathrm{V}_{\mathrm{s}}$ is the shear wave velocity after the decline of the shear modulus due to soil nonlinearity and related to the initial shear wave velocity $\mathrm{V}_{\mathrm{s}}$ by:

$$
\left(\frac{\mathrm{V}_{S}^{\prime}}{\mathrm{V}_{S}}\right)^{2}=\frac{1}{10}
$$

$1 / 10$ is a typical reduction factor of the shear modulus in a range of $1-10 \%$ on the strain dependent shear modulus curve.

The pile column system was modeled with 20 one dimensional (1D) beam elements. The pile tip is assumed to be pinned. Each node of the pile was connected to the soil mesh with soil-pile spring element. Details on the soil-pile spring could be found in Ozutsumi et al. ${ }^{10)}$.

The FE analyses were performed in two stages. In the first stage (self-weight analysis), the $i n$-situ stresses were initialized in the soil due to the own weight of the soil. Properties of pile and pile-soil interaction spring were set to be zero during this stage of analysis. During the second stage of analysis (seismic response analysis), the actual properties of soil, pile, and soil-pile interaction spring were assigned. The total mesh size was extended to a horizontal distance of 30-pile diameter to prevent spurious wave reflections at the boundaries. Moreover, tied lateral boundary approach (a simpler alternative to the boundary approach suggested by Zienkiewicz et al. ${ }^{14}$ ) that illustrated in Fig. 1. is used in the analysis. In this approach, the values of 
displacements, stresses, etc. are identical on both side boundaries. This condition is explicitly imposed in FLIP by an equivalent node concept.

The equation of motion of the soil-structure system is given as:

$$
\int[B]^{T}\left\{\sigma^{\prime}\right\} d v+[C]\{\underline{\dot{u}}\}+[M]\{\underline{u}\}=\int[B]^{T}\left\{\sigma_{s t}^{\prime}\right\} d v
$$

and

$$
\{\varepsilon\}=[B]\{\underline{u}\}
$$

where $\left\{\sigma^{\prime}\right\}$ and $\{\varepsilon\}$ are the effective stress and strain vectors, respectively. [C] and [M] are the damping and mass matrixes, respectively. $\{\underline{u}\}$ is the nodal displacement vector. $\left\{\sigma_{\text {st }}^{\prime}\right\}$ is the vector of effective stress due to gravity.

To solve the equation of motion step by step, the numerical integration was carried out by the Wilson- $\theta$ method $(\theta=1.4)$ using a time step of 0.01 seconds.

\subsection{Soil model}

The soil model used in this study consists of a multiple shear mechanism $^{11)}$ with the effective stress and strain vectors in plane strain condition expressed as

$$
\begin{gathered}
\left\{\sigma^{\prime}\right\}^{T}=\left\{\begin{array}{lll}
\sigma_{x}^{\prime} & \sigma_{y}^{\prime} & \tau_{x y}
\end{array}\right\} \\
\{\varepsilon\}^{T}=\left\{\begin{array}{lll}
\varepsilon_{x} & \varepsilon_{y} & \gamma_{x y}
\end{array}\right\}
\end{gathered}
$$

then the basic form of the constitutive relation is given by

$$
\left\{d \sigma^{\prime}\right\}=[D]\left(\{d \varepsilon\}-\left\{d \varepsilon_{p}\right\}\right)
$$

in which the term $\left\{d \varepsilon_{p}\right\}$ represents the volumetric strain increment due to dilatancy and is given by

$$
\left\{d \varepsilon_{p}\right\}^{T}=\left\{\begin{array}{lll}
d \varepsilon_{p} / 2 & d \varepsilon_{p} / 2 & 0
\end{array}\right\}
$$

The stiffness matrix is given by

$$
[D]=K\left\{n^{(0)}\right\}\left\{n^{(0)}\right\}^{T}+\sum_{i=1}^{I} G_{L / U}^{(i)}\left\{n^{(i)}\right\}\left\{n^{(i)}\right\}^{T}
$$

where the first term represents the volumetric mechanism with elastic tangent bulk modulus of soil skeleton $K$ and the direction vector given by

$$
\left\{n^{(0)}\right\}^{T}=\left\{\begin{array}{lll}
1 & 1 & 0
\end{array}\right\}
$$

and the second term represents the multiple shear mechanism without volume change. Each mechanism $i=1, \ldots, I$ represents a virtual simple shear mechanism, with each simple shear plane oriented at an angle $\theta_{i} / 2+\pi / 4$ relative to the $x$ axis. The tangential shear modulus $G^{(i)}{ }_{L / U}$ represents the hyperbolic stress strain relationship with hysteresis characteristics. The direction vectors for the multiple shear mechanism are given by

$$
\left\{n^{(i)}\right\}^{T}=\left\{\begin{array}{lll}
\cos \theta_{i} & -\cos \theta_{i} & \sin \theta_{i}
\end{array}\right\}(\text { For } i=1, \ldots \ldots, I)
$$

$$
\begin{array}{ll}
\theta_{i}=(i-1) \Delta \theta & (\text { For } i=1, \ldots, I) \\
\Delta \theta=\pi / I & (\text { For } i=1, \ldots \ldots, I)
\end{array}
$$

The loading (L) and unloading (U) for shear mechanism are separately defined for each mechanism by the sign of $\left\{n^{(i)}\right\}^{\mathrm{T}}\{d \varepsilon\}$. Each tangent modulus $G^{(i)}{ }_{L / U}$ depends on the present state and the history of each virtual simple shear strain $\gamma^{(i)}$. The virtual shear stress $q^{(i)}$ is introduced as the shear resistance variable to be defined per unit angle $\theta$ for mechanism $i$. When the inherent soil fabric is assumed to be isotropic, the virtual simple shear mechanism is defined by a hyperbolic relation under a constant confining stress as

$$
q^{(i)}=\frac{\gamma^{(i)} / \gamma_{v}}{1+\left|\gamma^{(i)} / \gamma_{v}\right|} q_{v}
$$

where $q_{v}$ and $\gamma_{\mathrm{v}}$ are the parameters for defining the hyperbolic relationship and called the virtual shear strength and virtual reference strain, respectively. The virtual tangent shear modulus are obtained for the initial loading as

$$
G_{L / U}^{(i)}=\frac{\gamma^{(i)} / \gamma_{v}}{\left(1+\left|\gamma^{(i)} / \gamma_{v}\right|\right)^{2}} \frac{q_{v}}{\gamma_{v}} \Delta \theta
$$

The relationships between the parameters $q_{v}$ and $\gamma_{\mathrm{v}}$ and the macroscopic shear strength $\tau_{m}$ and shear modulus $G_{m}$ can be written as

$$
\begin{aligned}
G_{m} & =\frac{q_{v}}{\gamma_{v}} \sum_{i=1}^{I} \sin ^{2} \theta_{i} \Delta \theta \\
\tau_{m} & =q_{v} \sum_{i=1}^{I} \sin ^{2} \theta_{i} \Delta \theta
\end{aligned}
$$

The shear modulus $G_{m}$ corresponding to effective mean stress $\sigma_{m}^{\prime}$ is related to the initial shear modulus $G_{m a}$ corresponding to initial effective mean stress $\sigma_{m a}^{\prime}$ as 


$$
G_{m}=G_{m a}\left(\frac{\sigma_{m}^{\prime}}{\sigma_{m a}^{\prime}}\right)^{m_{G}}
$$

where $\mathrm{m}_{\mathrm{G}}$ is a parameter that controls the shear modulus distribution with the depth. If $\mathrm{m}_{\mathrm{G}}=0$, the shear modulus will be constant with the depth and if $\mathrm{m}_{\mathrm{G}}=0.5$, the shear modulus will be proportional to the square root of the depth as shown in Fig. 2.

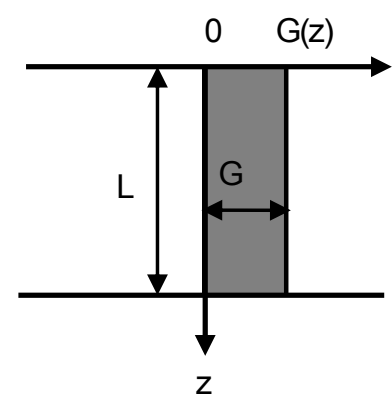

(a)

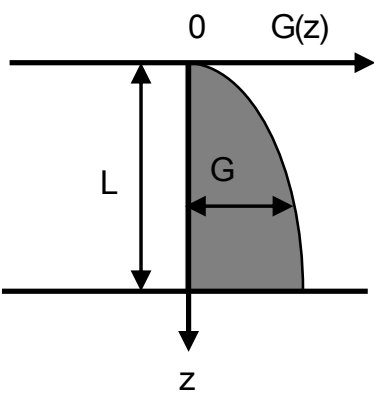

(b)
Fig. 2 Soil profiles studied in the analysis: (a) $m_{G}=0$ and (b) $\mathrm{m}_{\mathrm{G}}=0.5$

The shear strength is related to the angle of internal friction $\phi_{f}$ and cohesion c as

$$
\tau_{m}=c \cos \phi_{f}+\sigma_{m}^{\prime} \sin \phi_{f}
$$

Parameters for dry sand used in the current FE analysis are shown in Table 1. The bulk modulus of the soil skeleton $K$ was determined assuming a Poisson's ratio $v$ of 0.4 . The initial shear wave velocity $\left(\mathrm{V}_{\mathrm{s}}\right)$ is calculated using

$$
G_{m a}=\rho V_{s}^{2}
$$

and found to be $=200 \mathrm{~m} / \mathrm{s}$.

Table 1 Model parameters for soil elements.

\begin{tabular}{cccccc}
\hline $\begin{array}{c}\text { Density, } \rho \\
3 \\
(\mathrm{~m})\end{array}$ & $\begin{array}{c}G_{m a} \\
(\mathrm{kPa})\end{array}$ & $v$ & $\begin{array}{c}\sigma_{\operatorname{ma}}^{\prime} \\
(\mathrm{kPa})\end{array}$ & $\begin{array}{c}\phi_{f} \\
(\mathrm{deg})\end{array}$ & $H_{\max }$ \\
\hline 1.5 & $6.0 \times 10^{4}$ & 0.40 & 230 & 38 & 0.24 \\
\hline
\end{tabular}

\subsection{Pile Column System}

Linear elastic beam elements with three degrees of freedom per node are used for modeling pile and column. Normal force, shear force, and bending moment of each element are obtained directly from the used program.

It is assumed that the pile has a circular cross-section of diameter $D_{p}=1.5 \mathrm{~m}$, length $\mathrm{L}_{\mathrm{p}}=30 \mathrm{~m}$ (equal to the thickness of the soil stratum L), the pile slenderness ratio
$\left(\mathrm{L}_{\mathrm{p}} / \mathrm{D}_{\mathrm{p}}\right)=20$, Young's modulus $\mathrm{E}_{\mathrm{p}}=65 \mathrm{GPa}$ (i.e. $\mathrm{E}_{\mathrm{p}} / \mathrm{E}_{\mathrm{s}}=$ 1000 ), Poisson's ratio $v_{\mathrm{s}}=0.3$, and mass density $\rho_{\mathrm{p}}=2.5$ $\mathrm{t} / \mathrm{m}^{3}$. Same material properties were assigned for the column with only a change in column Young's modulus $\left(\mathrm{EI}_{\text {coloumn }} / \mathrm{EI}_{\text {pile }}=1.85\right)$.

Although the current paper is limited to the linear behavior of the pile and the structure, the non-linear behavior could be investigated using non-linear beam elements available in FLIP element library.

\subsection{Properties of the SDOF Structure}

The structural mass $\left(\mathrm{m}_{\mathrm{st}}\right)$ and height $\left(\mathrm{H}_{\mathrm{st}}\right)$ were set to be 100 $\mathrm{Mg}$ and $10 \mathrm{~m}$, respectively leading to a fixed base fundamental frequency of $8.4 \mathrm{~Hz}$ and a wave parameter $\sigma^{15)}$

$$
\sigma=\frac{V_{s}}{f_{\text {st.fixed }} \cdot H_{s t}}
$$

equal to 2.35 . The wave parameter may be looked upon as a measure of the relative stiffness of the soil and the structure. Parameter $1 / \sigma$ is utilized as a normalized fundamental frequency of the structure ${ }^{4)}$. Rovithis et al. ${ }^{4)}$ concluded that SSI effects are stronger for higher values of $1 / \sigma$ (i.e., $1 / \sigma>0.1$ ).

\section{Numerical Tool Validation}

Before proceeding to the non-linear analysis effects, a validation of SSPSI phenomenon assuming linear elasticity for soil is performed (i.e. a sample seismic signal is imposed at very low amplitude to ensure linear-elastic soil behavior). The shear modulus of soil is assumed to be constant with depth and a hysteretic damping ratio $\beta_{s}$ of 0.05 is used in the linear analysis.

\subsection{Free-Field Motion}

Elastic response time histories for free-field motion are derived at different frequencies of excitations. From these time histories, the amplitude of steady-state response is noted and normalized with respect to the amplitude of input bedrock. Thus, amplification of soil stratum is derived at different frequencies and compared with an established approach.

In Fig. 3, amplification of the soil stratum obtained using the present 2D FE model is compared with that obtained using a simple one-dimensional (1D) free-field analysis (in the frequency domain, Gazetas ${ }^{7}$ ) at different frequencies of excitation. Amplification for $1 \mathrm{D}$ analysis is given by:

$$
\frac{U_{f f}}{U_{g}}=\frac{1}{\cos (q L)} \quad \text { where } q=\frac{2 \pi f}{V_{s} \sqrt{\left(1+2 i \beta_{s}\right)}}
$$

where $\mathrm{L}=$ height of soil stratum; $f=$ frequency of excitation; $V_{s}$ $=\mathrm{S}$-wave velocity; $\beta_{s}=$ hysteretic damping ratio; $U_{g}$ and $U_{f f}=$ 
amplitude of input bedrock displacement and free-field ground displacement, respectively.

The comparison shows that there is an excellent agreement between the current FE method and the free-field analysis conducted by Gazetas ${ }^{7)}$ for all frequencies range covered in Fig. 3.

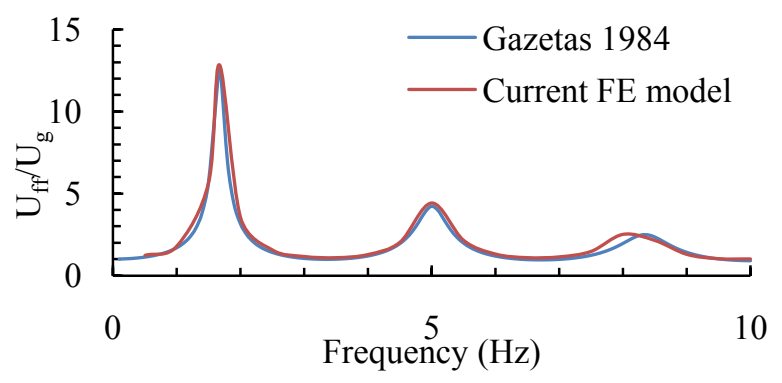

Fig. 3 Verification of the free-field amplification

\subsection{Kinematic Soil-pile Analysis}

A seismic S-wave propagating vertically in a soil layer without any foundation causes only horizontal displacements in the free-field soil. A cylindrical pile tends to diffract the incident seismic waves, modifying the free-field soil motion, so that the horizontal displacement of the pile head, $U_{p}$, will be different from the free-field surface motion $U_{\mathrm{ff}}$, and a rotation $\phi_{\mathrm{p}}$ of the pile head will take place. This type of interaction between piles and soils is called kinematic interaction. This kinematic effect is usually assessed in terms of the kinematic interaction factors $I_{u}$ and $I_{\phi}$, which are defined, respectively, as the maximum steady-state translation and rotation of the pile head normalized by the corresponding maximum steady-state displacement of the free field soil surface as:

$$
I_{u}=\frac{U_{p}}{U_{f f}} \text { and } I_{\phi}=\frac{\phi_{p} \cdot D_{p}}{U_{f f}}
$$

Similar to free-field response, the elastic response time histories of pile head $\left(U_{p}\right.$ and $\left.\phi_{p}\right)$ are derived at different frequencies of excitations. From these time histories, the amplitude of steady-state response is noted and used to obtain the kinematic interaction factors using Eq. (24). The kinematic interaction factors obtained by this study were compared to established results from the literature ${ }^{4)}{ }^{8}$. Both fixed and free conditions at pile head were considered.

For a fixed-head pile, the analytical solution for $I_{u}$ is given by the expression ${ }^{16)}$.

$$
I_{u}=\Gamma=\frac{k+i w c}{E_{p} I_{p}\left(\mathrm{q}^{4}+4 \lambda^{4}\right)}
$$

which was obtained through a properly calibrated dynamic Winkler model. The corresponding kinematic interaction factors for a free head pile are (Nikolaou et al. $\left.{ }^{17)}\right)$ :

$$
\begin{gathered}
I_{u}=\Gamma\left[1+\frac{1}{2}\left(\frac{\mathrm{q}}{\lambda}\right)^{2}\right] \\
I_{\phi}=\Gamma \frac{\mathrm{q}^{2} D_{p}}{\lambda}
\end{gathered}
$$

in the above equations, $(\mathrm{k}+\mathrm{iwc})$ denotes the so-called dynamic impedance of the Winkler bed, with $w$ being the cyclic vibrational frequency and $i$ the imaginary unity; $\mathrm{q}=w / \mathrm{V}_{\mathrm{s}}$ is the wave number of the harmonic $\mathrm{SH}$ waves in the soil; $\lambda$ is the well-known Winkler wave number:

$$
\lambda=\left\{\frac{k+i w c-m w^{2}}{4 E_{p} I_{p}}\right\}^{\frac{1}{4}}
$$

Based on the comparative results shown in Fig. 4 that shows the variation of kinematic interaction factors with the normalized frequency $\left(\mathrm{a}_{0}=\mathrm{w}^{*} \mathrm{D}_{\mathrm{p}} / \mathrm{V}_{\mathrm{s}}\right)$, it is established that the adopted FE model captures well the variation of kinematic pile response in wide range of frequency.
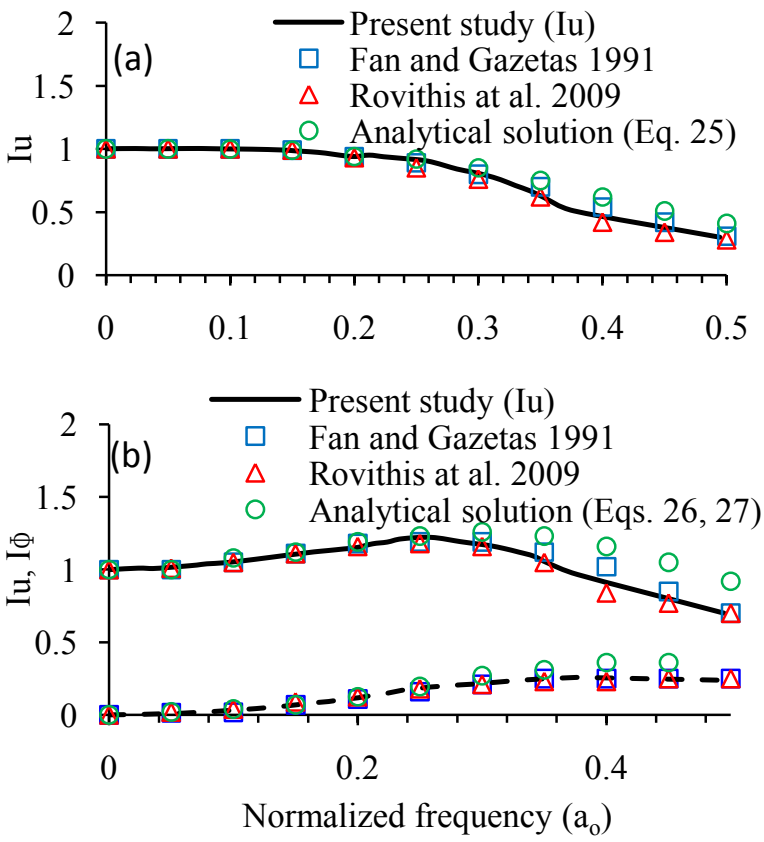

Fig. 4 Kinematic soil-pile interaction for: (a) fixed head and (b) free head conditions

\subsection{Kinematic Pile Bending Moment}

The verification process continues to include the kinematic pile bending moment arising from the passage of seismic waves 
through soil layer. The pile bending moments were normalized to the amplitude of bedrock acceleration ${ }^{4), 8)}$.

$$
M_{n o r}=\frac{M}{\rho_{p} D_{p}^{4} \ddot{U}_{g}}
$$

where $\ddot{\mathrm{U}}_{\mathrm{g}}$ is the amplitude of the bedrock acceleration $\ddot{\mathrm{U}}_{\mathrm{g}}=w^{2} \mathrm{U}_{\mathrm{g}}$. Figure 5 presents the distribution of amplitudes of normalized steady state bending moments for the free and fixed pile head conditions at the first two fundamental frequencies of the soil deposit $f_{1}$ and $f_{2}$ calculated using the present FE method compared to those obtained by Rovithis et al. ${ }^{4}$ with the same soil and pile conditions. Figure 5 implies that the current FE analysis reasonably reproduced normalized pile bending moment agree well with the previous established one by Rovithis et al. ${ }^{4)}$ for different pile head conditions and different fundamental frequencies of soil as well.

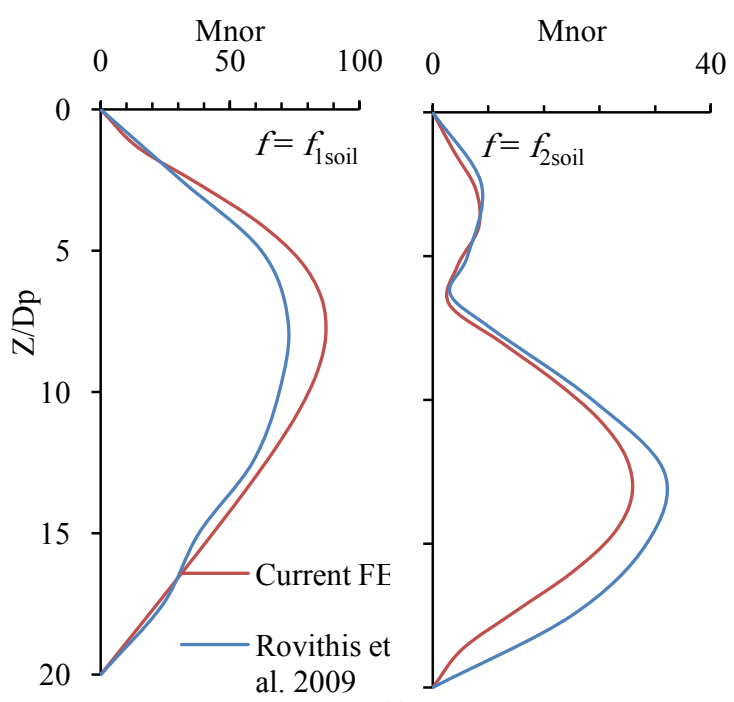

(a)

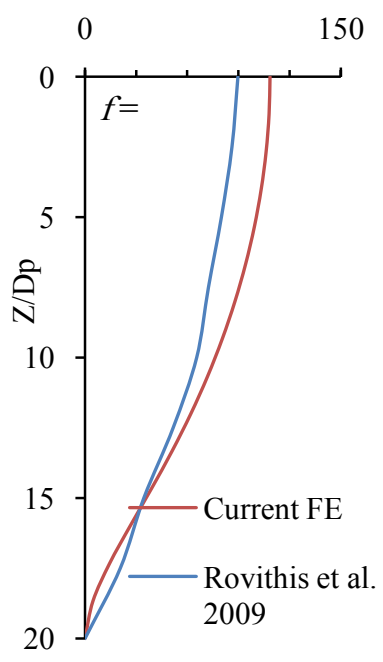

0

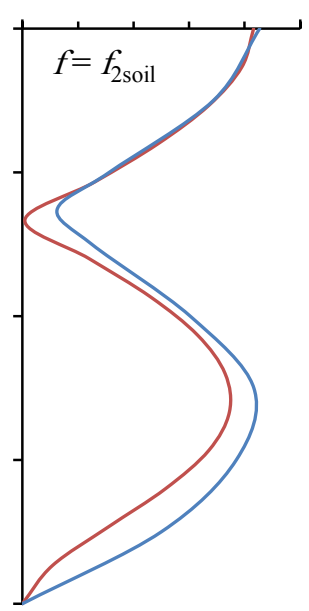

(b)

Fig. 5 Normalized bending moments at the first two frequencies of the soil deposits: (a) free head pile; (b) fixed head pile

\subsection{Dynamic Characteristics of the Coupled SPS System}

In this step, a concentrated mass attached to the structural column is introduced to the model. The verification process in this stage focuses on evaluating the effective natural $\left(f_{\text {sSI }}\right)$. The effective natural frequency $\left(f_{\text {SSI }}\right)$ of the coupled system is defined as the frequency at which the ratio $\left(\mathrm{U}_{\mathrm{s}} / \mathrm{U}_{\mathrm{ff}}\right)$ is maximized, where $\mathrm{U}_{\mathrm{s}}$ is the structure mass displacement. Verification was performed by comparing the effective natural period of the system $\left(T_{\mathrm{SSI}}\right)$ calculated using the current FE model at different values of parameter $1 / \sigma$ with the available analytical solutions as well as the 3D FE analysis by Rovithis et al. ${ }^{4}$. The available analytical procedures include:

$$
\begin{aligned}
& \text { Solution by Veletsos and Meek }{ }^{15)} \text { : } \\
& \qquad T_{S S I}=T_{s t, \text { fixed }} \sqrt{1+\frac{K_{s t}}{K_{x}}\left(1+\frac{K_{x} H_{s t}^{2}}{K_{\theta}}\right)}
\end{aligned}
$$

where $\mathrm{T}_{\text {st fixed }}$ and $\mathrm{K}_{\mathrm{st}}$ represent the fixed-base natural period and stiffness of the structure. $\mathrm{K}_{\mathrm{x}}$ and $\mathrm{K}_{\theta}$ denote the frequency-dependent foundation stiffness in translational and rocking oscillations, respectively.

(2) Rigorous analytical solution by Maravas et al. ${ }^{18)}$ :

$$
\begin{aligned}
& T_{S S I}= \\
& T_{s t, \text { fixed }} \sqrt{\frac{K_{s t r}}{K_{x}}\left(\frac{1+4 \zeta^{2}}{1+4 \zeta_{x}^{2}}\right)+\frac{K_{s t r} H_{s t r}^{2}}{K_{\theta}}\left(\frac{1+4 \zeta^{2}}{1+4 \zeta_{\theta}^{2}}\right)+\left(\frac{1+4 \zeta^{2}}{1+4 \zeta_{s t r}^{2}}\right)}
\end{aligned}
$$

where $\zeta_{\text {str }}$ and $\zeta$ represent the damping ratio of the fixed-base and flexibly supported structure, respectively, and $\zeta_{x}, \zeta_{\theta}$ the damping ratios in swaying and rocking of the foundation.

Comparison of the current FE model results against the aforementioned equations as well as the results obtained by Rovithis et al. ${ }^{4}$ ) is illustrated in Fig. 6 . Figure 6 presents the ratio of the effective natural frequency of the system to the natural frequency of the fixed-base structure $\left(f_{\text {ssl }} / f_{\text {str. fixed }}\right)$ against the parameter $(1 / \sigma)$. A very good agreement between the numerical results and both the rigorous analytical solution of Maravas et al. ${ }^{18)}$ and the 3D analysis of Rovithis et al. ${ }^{4}$ is evident confirming the accuracy of the FE model.

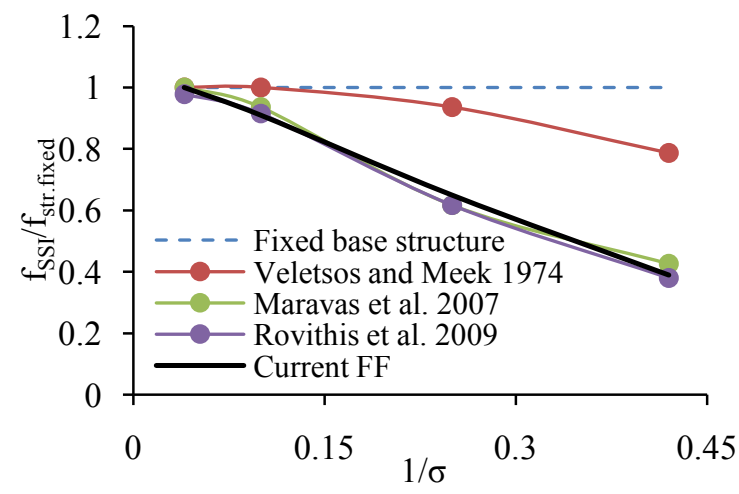

Fig. 6 Ratio of the effective natural frequency of the system to the structural fixed-base frequency against parameter $1 / 0$ 


\section{Effect of Soil Profile and Soil Nonlinearity}

The effects of soil profile and material nonlinearity of the soil are a major concern. It is anticipated that the soil stratum plays a significant role in the response of the SSPSI system.

\subsection{Free-Field Response}

The effect of soil profile on the free-field response is studied by changing the value of parameter $\mathrm{m}_{\mathrm{G}}$ in Eq. (19) as stated earlier. The effect of nonlinearity of soil is also studied by changing the amplitude of the input acceleration (A). Figure 7 compares the variation of the free-field amplification with respect to bed rock motion versus frequencies of the input motion. The second and the third natural shear frequencies of the soil layer get closer to the first natural shear frequency as the soil shear modulus distribution with depth become not uniform (inhomogeneous), in agreement with the results obtained by Gazetas $^{7}$. A substantial increase is observed in the peak amplification at the first, the second, and the third natural frequencies as the soil become inhomogeneous. Figure 7 shows also the fundamental periods lengthening due to soil nonlinearity and the substantial reduction in the peak amplification accompanied with these periods lengthening.

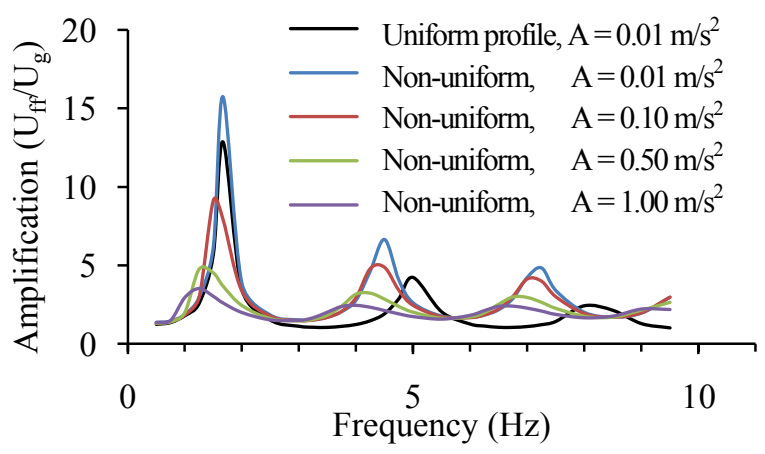

Fig. 7 Influence of soil profile and soil non-linearity on the free-field response

The strain level of the soil might be different with the change of the frequency of the input motion, even if the peak acceleration was constant. Figure 8 shows the variation of the shear strain of the ground with the frequency of input motions at constant values of the amplitude of input accelerations. As expected, a substantial increase is observed in the peak amplification of soil shear strain at the first, the second, and the third natural frequencies with the increase of the amplitude of the input motion.

\subsection{Kinematic Soil-pile Interaction}

The influence of soil profile on kinematic interaction factors for both fixed and free head conditions is portrayed in Fig. 9. Figure 9 declares that, in the frequency range studied $(f=$ $0.5-10.5 \mathrm{~Hz}$ ), the filtering by a pile of the high frequency components of the base excitation may be substantially greater with inhomogeneous than with homogeneous soil deposits. At the same time, the rotational components of motion may also be stronger in the inhomogeneous deposits at high frequency components of the base excitation but weaker at lower ones. On the other hand, the effect of non-linear soil properties seems to be not significant for both interaction factors components.

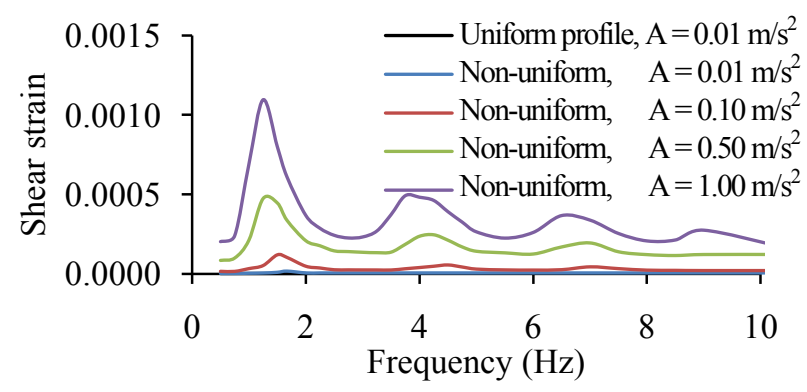

Fig. 8 Shear strain variation with frequency and amplitude of input motion.

\subsection{Coupled Soil-pile-structure Interaction}

As stated in section 2, the effective natural frequency $\left(f_{\text {SSI }}\right)$ is defined as the frequency at which the ratio $\left(\mathrm{U}_{\mathrm{s}} / \mathrm{U}_{\mathrm{ff}}\right)$ is maximized. The effect of soil profile and material nonlinearity of soil on the effective natural frequency of a coupled soil-pile-structure system $(1 / \sigma=0.42)$ is shown in Fig. 10. The fixed base fundamental frequency of the structure is also plotted as a reference. Figure 10 shows the strong effect of soil-pile-structure interaction resulting in a significant reduction of $f_{\mathrm{SSI}}$ with respect to the natural frequency of the structure under fixed base conditions. Figure 10 also illustrates a reduction in $f_{\text {SSI }}$ corresponding to the change in soil profile from homogeneous to inhomogeneous soil profile. A reduction in $f_{\mathrm{SSI}}$ due to soil nonlinearity associated with substantial decrease in the peak amplification could be observed from Fig. 10.

\subsection{Combined Kinematic and Inertial Pile Response.}

Figure 11 plots the ratios of the pile-head to free-field displacement $\left(\mathrm{U}_{\mathrm{p}} / \mathrm{U}_{\mathrm{ff}}\right)$ under the combined effect of kinematic and inertial interaction for both homogeneous and inhomogeneous soil profiles at a low amplitude of input motion (input acceleration $=0.01 \mathrm{~m} / \mathrm{s}^{2}$ ) to ensure linear behavior. Figure 11 plots also the ratios of $\left(\mathrm{U}_{\mathrm{p}} / \mathrm{U}_{\mathrm{ff}}\right)$ correspond to inhomogeneous soil profile and at different amplitudes of input motions ranging from 0.01 to $1.0 \mathrm{~m} / \mathrm{s}^{2}$ input acceleration to investigate the effect of soil nonlinearity on the pile response under the combined action of kinematic and inertial interactions. It is worth to note that at least for all cases included in Fig. 11, the ratio $\mathrm{U}_{\mathrm{p}} \mathrm{U}_{\mathrm{ff}}$ seems to be dominated by two discrete frequencies: a lower frequency where the pile-head motion is amplified and a higher one where the response is suddenly de-amplified with respect to 
free-field motion. This variation pattern has been observed in transient response analyses using real earthquake motions of a pile-supported structure ${ }^{19)}$ as well as analytical studies of soil-pile-structure system base on coupled ${ }^{4)}$ and uncoupled ${ }^{20)}$ SSPSI.
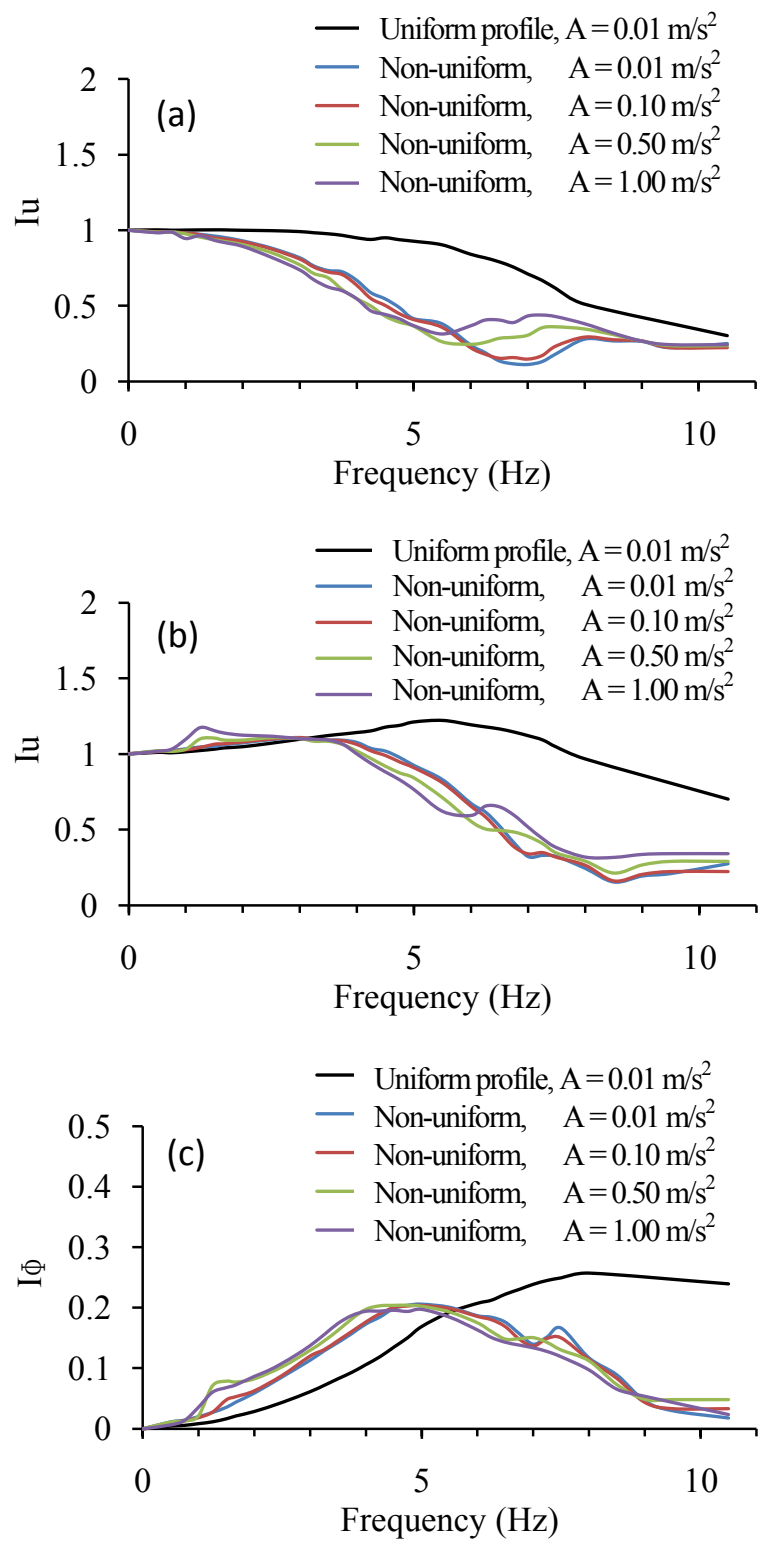

Fig. 9 Effect on the interaction factors: (a) $\mathrm{I}_{\mathrm{u}}$ (Fixed head); (b) $\mathrm{I}_{\mathrm{u}}$ (free head); and (c) $\mathrm{I}_{\phi}$ (free head)

As observed by Rovithis et al. ${ }^{4)}$, pile-head motion is substantially amplified at the effective natural frequency $f_{\mathrm{SSS}}$, where the response of the pile-structure system is maximized. A pseudo-natural frequency $\left(f_{\mathrm{pSSI}}\right)$ is introduced by Rovithis et al. ${ }^{4)}$ (using linear 3D FE analysis) as the frequency where the pile-head motion is minimized relative to the free-field soil surface motion. As shown in Fig. 11 there is some reduction in $f_{\mathrm{pSS}}$ as the soil become inhomogeneous. In contrast to the clear effect of soil nonlinearity on $f_{\mathrm{SSb}} f_{\mathrm{pSSI}}$ seems to be not affected by soil nonlinearity.

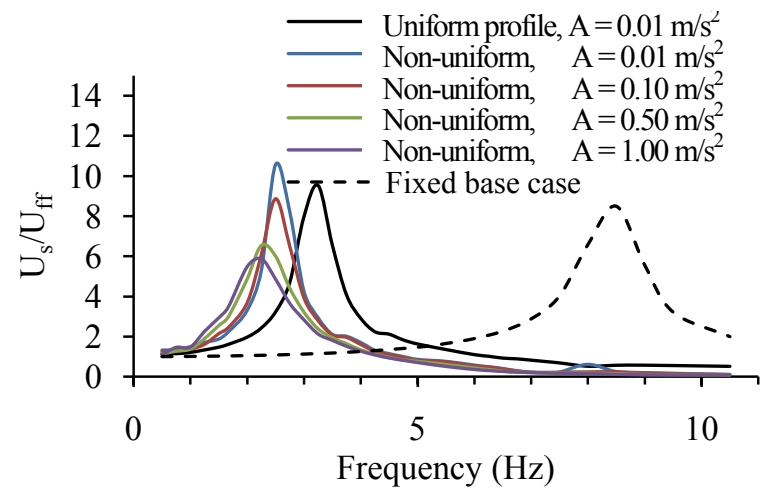

Fig. 10 Effect on effective natural frequency $\left(f_{\text {SSI }}\right)$ of the soil-pile-structure system: $1 / \sigma=0.42$

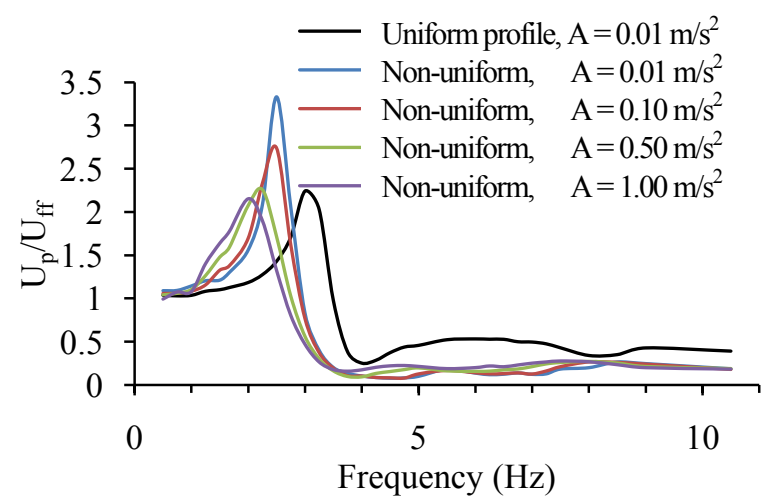

Fig. 11 Effect on pile head to free field displacement response of the coupled system

\section{Dynamic Pile Moment: Relative Contributions of Kinematic and Inertial Interaction.}

To investigate the role of the frequency content of the input motion as well as the relative contributions of kinematic and inertial interaction on dynamic pile bending, pile bending moments profiles calculated at frequencies content of input motions closed to both natural frequencies of the soil stratum and effective natural frequency of the coupled soil-pile-structure system are plotted and compared to the corresponding kinematic moments for both free and fixed pile head conditions as shown in Fig. 12. Figure 12 (a), (b), (c), and (d) present dynamic pile bending obtained at $1.0 \mathrm{~m} / \mathrm{s}^{2}$ input acceleration and 1.25, 2.25, 3.75 , and $6.75 \mathrm{~Hz}$ respectively.

When the frequency content of the bedrock excitation is closed to the first fundamental frequency of soil $(1.25 \mathrm{~Hz})$ as shown in Fig. 12(a), the maximum pile bending moment occurs near the ground surface (pile head) and it corresponds to the fixed head pile case (kinematic interaction). Figure 12(a) declares that SSPSI has minor effect on pile bending and the 
kinematic interaction is predominant. Figure 12(b) shows the results at $2.0 \mathrm{~Hz}$ which corresponds to the effective natural frequency of the coupled system (see Fig. 11). The maximum pile bending moment occurs near the ground surface and it corresponds to the coupled soil-pile-structure system. Figure 12(b) declares that soil-pile-structure interaction has a major effect on pile bending. Figure 12(c) shows a substantial reduction in pile bending under the combined action of kinematic and inertial interaction when the frequency content of the input motion is closed to $f_{\mathrm{pSSI}}=3.75 \mathrm{~Hz}$. This reduction in pile bending may be important in pile design. It is worth to note that, although this significant reduction, the maximum pile bending still corresponds to the combined action of kinematic and inertial interaction and it occurs close to ground surface. When the dominate frequency of the input motion excites higher soil modes, kinematic effects dominate the response and significant bending moment are generated at greater depths as shown in Fig. 12(d).

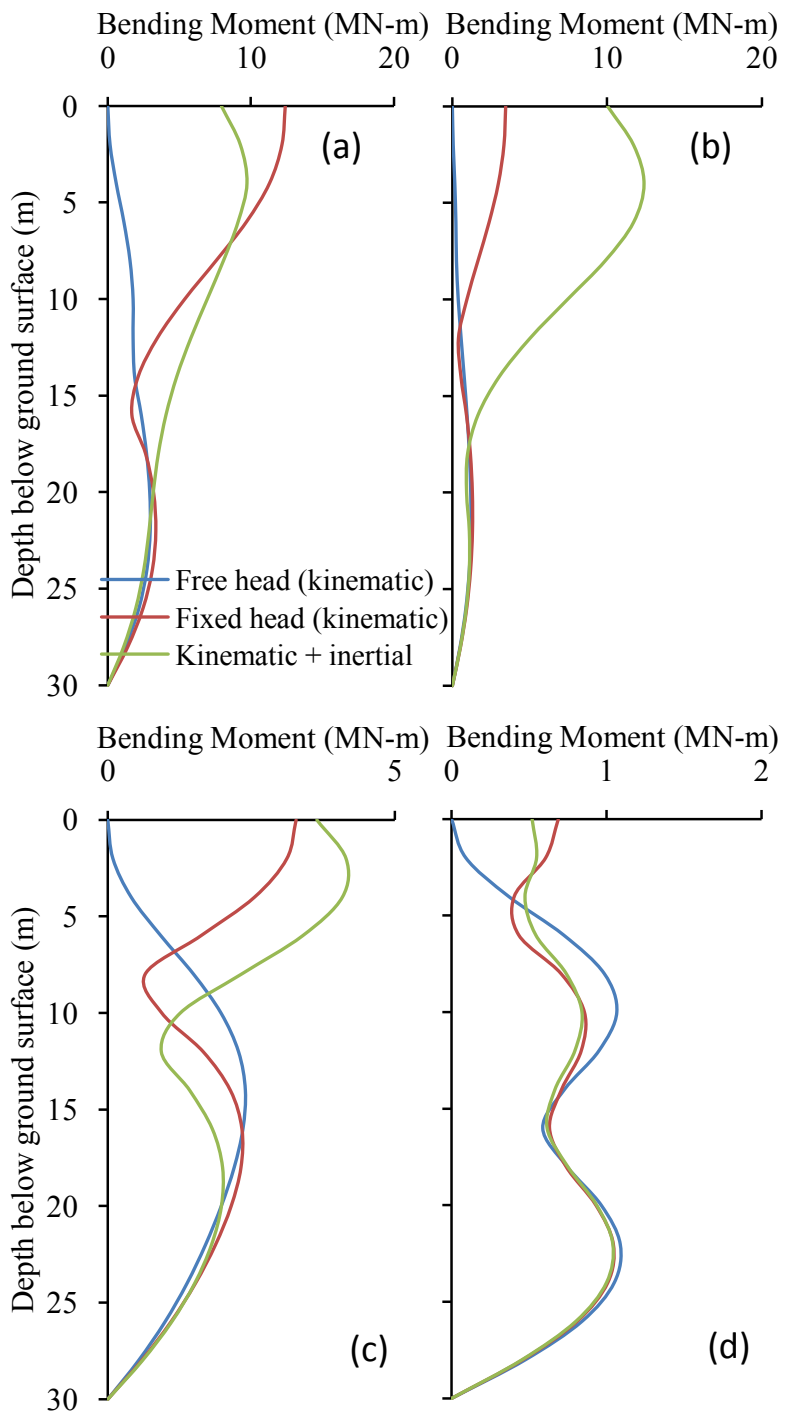

Fig. 12 Pile bending profile at different frequencies: (a) $1.25 \mathrm{~Hz}$, (b) $2.0 \mathrm{~Hz}$, (c) $3.75 \mathrm{~Hz}$, and (d) $6.75 \mathrm{~Hz}$.

\section{Conclusions}

The non-linear response of coupled soil-pile-structure systems to seismic loading was parametrically studied in the frequency domain using 2D FE. The interaction between the pile and the surrounding soil in 3D type was idealized in the 2D analysis using a soil-pile interaction spring with a hysteretic non-linear load displacement relationship. The study focuses on the effects of soil profile and material nonlinearity of the soil on the kinematic and inertial interaction as affected by the frequency content of input motion. Of the findings of this study, the following conclusions can be drawn:

(1) Kinematic soil-pile interaction: the filtering of the pile to the high frequency components of the base excitation is substantially greater with inhomogeneous than with homogeneous soil deposits. On the other hand the effect of non-linear soil behavior seems to be not significant.

(2) Coupled kinematic and inertial interaction: there is a significant reduction in the effective natural frequency due to soil inhomogeneity as well as soil nonlinearity.

(3) In contrast to the clear effect of soil nonlinearity on $f_{\mathrm{SSI}}, f_{\mathrm{pSSI}}$ seems to be not affected by it.

(4) Structural vibrations tend to impose large bending moments on or near to the pile head, when the frequency of excitation is close to the effective natural frequency of the system. On the contrary, when the input motion excites higher soil modes, strong kinematic effects are mobilized and generate significant pile bending at greater depths.

\section{References}

1) Kagawa, T. and Kraft, L., Seismic P-Y responses of flexible piles, Journal of Geotechnical Engineering, ASCE, 106(8), 899-918, 1980.

2) Allotey, N. and El Naggar, M.H., Generalized dynamic Winkler model for nonlinear soil-structure interaction analysis, Canadian Geotechnical Journal, 45(4), 560-573, 2008.

3) Cai, Y.X., Gould, P.L. and Desai, C.S., Numerical implementation of a 3-D nonlinear seismic S-P-S-I methodology, In Seismic Analysis and Design for Soil-Pile-Structure Interactions, Prakash S (ed.), Geotechnical Special Publication, 70, ASCE, 96-110, 1997.

4) Rovithis, E., Pitilakis, K. and Mylonakis, G., Seismic analysis of coupled soil-pile-structure systems leading to the definition of a pseudo-natural SSI frequency, Soil Dynamics and Earthquake Engineering, 29(6), 1005-1015, 2009.

5) Kattis, S.E., Polyzos, D. and Beskos, D.E., Vibration isolation by a row of piles using a 3-D frequency domain 
BEM, International Journal for Numerical Methods in Engineering, 64(5), 713-728, 1999.

6) Padrón, J.A., Aznárez, J.J. and Maeso, O., BEM-FEM coupling model for the dynamic analysis of piles and pile groups, Engineering Analysis with Boundary Elements, 31(6), 473-484, 2007.

7) Gazetas, G., Seismic response of end bearing single piles. Soil Dynamics and Earthquake Engineering, 3(2), 82-93, 1984.

8) Fan, K., Gazetas, G., Kaynia, A., Kausel, E. and Ahmad, S., Kinematic seismic response of single piles and pile groups. Journal of Geotechnical Engineering, 117(12), 1860-1879, 1991.

9) Kaynia, A. M. and Mahzooni, S., Forces in pile foundations under seismic loading. Journal of Engineering Mechanics, 122(1), 46-53, 1996.

10) Ozutsumi, O., Tamari, Y., Oka, Y., Ichii, K., Iai, S. and Umeki, Y., Modeling of soil-pile interaction subjected to soil liquefaction in plane strain analysis, Proc. of the 38th Japan National Conference on Geotechnical Engineering, Akita,1899-1900, 2003.

11) Iai, S., Matsunaga, Y. and Kameoka, T., Strain space plasticity model for cyclic mobility, Soils and Foundations, 32(2), 1-15, 1992.

12) Hussien, M. N., Tobita, T. and Iai, S., Nonlinear seismic finite element analysis of soil-pile-superstructure interaction, Journal of Applied Mechanics, JSCE, 13, 601-609, 2010.

13) Matthees, W. and Magiera, G., A sensitivity study of seismic structure-soil-structure interaction problems for nuclear power plants. Nuclear Engineering and Design 73(3),
343-363, 1982.

14) Zienkiewicz, O.C., Bicanic, N. and Shen, F.Q., Earthquake input definition and the transmitting boundary conditions. In Advances in Computational Nonlinear Mechanics, Springer-Verlag, 109-138, 1988.

15) Veletsos, A.S. and Meek, J.W., Dynamic behavior of building-foundation systems. Earthquake Engineering and Structural Dynamics, 3, 121-38, 1974.

16) Makris, N. and Gazetas, G., Dynamic pile-soil-pile interaction, part II: Lateral and seismic response. Earthquake Engineering and Soil Dynamics, 21(2), 145-62, 1992.

17) Nikolaou, S., Mylonakis, G., Gazetas, G. and Tazoh, T., Kinematic pile bending during earthquakes: analysis and field measurements. Geotechnique, 51(5), 425-40, 2001.

18) Maravas, A., Mylonakis, G. and Karabalis, D., Dynamic characteristics of simple structures on piles and footings. In: Proceedings of the 4th international conference on earthquake geotechnical engineering. Thessaloniki, Greece, paper no. 1672, 2007.

19) Ohata, T., Uchiyama, S., Niwa, M. and Ueno, K. Earthquake response characteristics of structure with pile foundation on soft subsoil layer and its simulation analysis. In: Proceedings of the 7th world conference on earthquake engineering. Vol. 3. Istanbul, Turkey, 1980.

20) Mylonakis, G., Nikolaou, A. and Gazetas, G. Soil-pile-bridge seismic interaction: kinematic and inertial effects. PartI: Soft soil. Earthquake Engineering and Structural Dynamics; 26: 337-59, 1997.

(Received: March 8, 2011) 